\title{
The Role of Intravenous Iron in the Treatment of Anemia Associated with Cancer and Chemotherapy
}

\author{
George M. Rodgers a Jeffrey A. Gilreath ${ }^{b}$ \\ ${ }^{a}$ Division of Hematology and Hematologic Malignancies, University of Utah Health Sciences Center, Salt Lake City, \\ UT, USA; ${ }^{b}$ Department of Pharmacotherapy, University of Utah College of Pharmacy, Salt Lake City, UT, USA
}

\section{Keywords}

Functional iron deficiency · Chemotherapy-induced anemia $\cdot$ Intravenous iron $\cdot$ Cancer

\begin{abstract}
Cancer-related anemia (CRA) is a commonly occurring problem for patients with cancer regardless of whether they are receiving treatment with chemotherapy or immunotherapy. It may result from one or more processes (decreased production, increased destruction, or increased loss of red blood cells, RBC). Perturbations in iron availability form the primary basis for anemia in many patients with cancer-related anemia. Functional iron deficiency (FID) anemia is a condition in which the patient has adequate or increased iron stores, but this iron pool is not available for erythropoiesis. Erythropoiesis-stimulating agents (ESAs) were the original treatment for FID; over time, however, if the supply of iron cannot keep pace with increased RBC synthesis driven by ESAs, FID may eventually lead to the lack or loss of ESA responsiveness. Subsequent clinical trials reported that intravenous (IV) iron could enhance the erythropoietic response to ESAs. This chapter reviews the pathogenesis of FID and summarizes the literature on the treatment of cancer- and chemotherapy-induced anemia. Clinical trials using IV iron with or without ESAs are reviewed in addition to the currently available
\end{abstract}

IV iron products. The consensus conclusions from these trials, as well as guideline recommendations, support the use of IV iron in these patients to enhance ESA responsiveness, decrease ESA dosage, and reduce RBC transfusions. Little data have been published on the long-term safety of IV iron or its impact on tumor growth. This paper also briefly explores novel approaches for the treatment of FID anemia, which has relevance in treating not only cancer patients but also patients with benign inflammatory disorders.

(c) 2019 S. Karger AG, Basel

\section{Introduction}

Despite the increasing use of targeted therapies in cancer patients, chemotherapy remains a mainstay of cancer treatment. Consequently, anemia remains a common, expected complication in patients receiving chemotherapy [1]. In addition to the myelosuppressive effects of chemotherapy, other cancer-associated conditions that contribute to the decreased production of red blood cells (RBCs) and lead to anemia include: renal disease associated with erythropoietin deficiency, tumor involvement within the bone marrow, possible vitamin deficiencies, and, perhaps most importantly, functional iron deficiency (FID) [1]. Synonyms for FID include "anemia of chronic disease" and "anemia of

\section{KARGER}

(c) 2019 S. Karger AG, Basel

E-Mail karger@karger.com

www.karger.com/aha
George M. Rodgers, MD, PhD

Division of Hematology and Hematologic Malignancies

University of Utah Health Sciences Center

Salt Lake City, UT 84132 (USA)

E-Mail George.Rodgers@hsc.utah.edu 
inflammation." FID is an important contributor to anemia not only in cancer patients but also in those with infectious or inflammatory disorders, such as inflammatory bowel disease and rheumatoid arthritis, for example.

Understanding the pathogenesis of FID is crucial to understanding the basis of anemia in cancer patients as well as in patients with benign inflammatory disorders. This paper summarizes the role of FID in cancer- and chemotherapy-associated anemia and reviews clinical trial results on treating chemotherapy-induced anemia (CIA) with a focus on the use of intravenous (IV) iron.

\section{Pathogenesis of FID}

Although the pathogenesis of FID has been elucidated over the past 10-20 years, it is still not fully understood [2]. Two key cells involved in the process of iron movement into and throughout the body are enterocytes of the gastrointestinal tract (responsible for absorbing dietary or supplemental oral iron) and macrophages of the reticuloendothelial system in the bone marrow (responsible for mobilization of iron stores used primarily for erythropoiesis). Two key proteins in this mechanism are ferroportin, an iron transport protein present in enterocytes and macrophages that controls cellular iron export, and hepcidin, an inflammatory response protein that regulates the ability of ferroportin to export iron. Figure 1 summarizes aspects of iron metabolism that relate to normal iron utilization and erythropoiesis, as well as the consequences of inflammation (due to benign or malignant conditions) that lead to FID. Known ligands, receptors, intracellular signaling pathways, and hormones regulate iron mobilization are included. Known constituents of the iron life cycle are listed in Figure 1.

In the normal, noninflamed state (low hepcidin concentrations), dietary or supplemental oral iron is ingested; enterocytes in the proximal small bowel absorb iron, and that iron is transported via enterocyte ferroportin to blood vessels where iron then becomes bound to serum transferrin and carried either to erythroblasts, bone marrow macrophages, or other tissues in the body such as the brain [2]. Iron is stored in spleen and marrow macrophages until needed for erythropoiesis, at which time iron is transported via macrophage ferroportin to serum transferrin and then to RBC precursors, where it is utilized to synthesize hemoglobin (Hb) (Fig. 1).

In the "inflamed" state of cancer or other inflammatory disorders, hepcidin synthesis by the liver is increased in response to cytokines such as interleukin (IL)-6. Ele- vated hepcidin levels result in inhibition of ferroportinmediated iron transport in both enterocytes and macrophages. Hepcidin binding to ferroportin leads to internalization and lysosomal destruction of the hepcidinferroportin complex with subsequent sequestration of iron within both enterocytes and macrophages (Fig. 1). The result of this hepcidin upregulation is decreased serum iron, decreased transferrin-bound iron, and a lack of available iron for erythropoiesis, despite adequate (or increased) iron stores, hence the name FID. The recently discovered hormone erythroferrone (nicknamed ERFE) is secreted by erythroblasts to decrease hepcidin and increase iron bioavailability when erythropoiesis is under stress (Fig. 1) [3].

Two important conclusions that can be drawn from this information are (a) oral iron may be ineffective in treating FID from any cause, since ingested iron may be sequestered in the enterocyte and not available for erythropoiesis, and (b) IV iron should be effective in overcoming the gastrointestinal and macrophage "iron block" of FID.

Based on the above pathogenesis information on FID, numerous clinical trials have been conducted that reported clinically useful information on how FID patients should be managed. We will first review the IV iron products that have been studied in CIA clinical trials; then, results of clinical trials using IV iron, with and without erythropoiesis-stimulating agents (ESAs), will be summarized.

\section{Iron Products with Efficacy in CIA}

Six IV products have been studied in CIA clinical trials and reported to be efficacious: low-molecular-weight iron dextran, iron sucrose, ferric gluconate, ferric carboxymaltose, ferumoxytol, and iron isomaltoside. Table 1 summarizes these products and their recommended dosing regimens. Fewer reported CIA patients have been treated with ferumoxytol or iron isomaltoside in clinical trials compared to those treated with the other products.

\section{Summary of CIA Clinical Trials Using IV Iron with ESAs}

Seven clinical trials in CIA patients using IV iron with ESAs versus ESA alone have been published as of February 2019. Table 2 summarizes these trials, IV iron prod-
Acta Haematol 2019;142:13-20 DOI: $10.1159 / 000496967$
Rodgers/Gilreath 


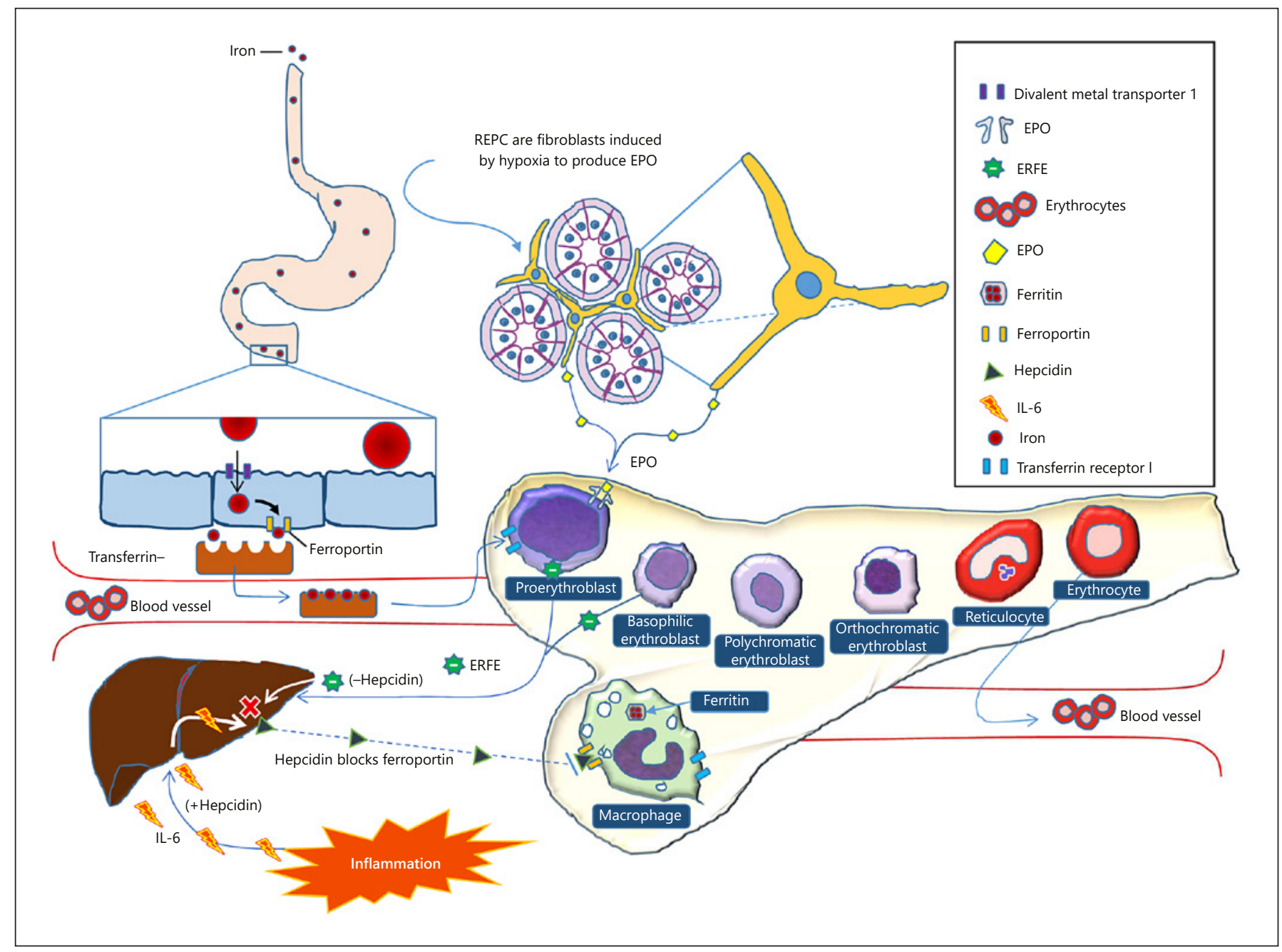

Fig. 1. Normal iron metabolism, erythropoiesis, and pathogenesis of functional iron deficiency. In the normal (noninflamed) state, dietary iron (or an oral iron supplement) is ingested. Iron is absorbed by enterocytes in the proximal small intestine via the divalent metal transporter 1 and transported to blood vessels via ferroportin, then to the bone marrow via transferrin. In the bone marrow, iron is taken up by macrophages where it is stored as ferritin until needed for erythropoiesis. At that time, stored iron is transferred via ferroportin to red blood cell (RBC) precursors for hemoglobin synthesis. Erythropoietin (EPO) is the physiologic regulator of RBC production. EPO is produced by fibroblasts in the kidney in response to hypoxia. In the "inflamed state" (lower

ucts used, numbers of CIA patients studied, and key responses. Of note, only clinical trials published as full articles, but not abstracts, are included in this analysis. At the time of original publication, 6 of 7 trials reported positive results, including improving the ESA response rate, allowance of a lower ESA dose, and decreased RBC transfusion requirement. Reanalysis of the data in the negative portion of Fig. 1), interleukin (IL)-6 is produced, inducing hepatic release of hepcidin. Hepcidin binds both enterocyte and macrophage ferroportin; the hepcidin-ferroportin complexes are internalized and degraded in lysosomes, resulting in an "iron block" inability of enterocyte- and macrophage-stored iron to be exported. (For figure simplicity, only hepcidin inhibition of macrophage ferroportin is shown.) This leads to reduced serum iron levels, increased storage of iron in bone marrow macrophages (i.e., ferritin), and the condition of functional iron deficiency. Erythroferrone (ERFE) is a negative regulator of hepcidin production. ERFE is produced by erythroblasts in response to EPO. REPC, renal EPOproducing cells. trial subsequently demonstrated a positive result when patients intolerant to doses above those that are routinely recommended were censored. Thus, all clinical trials utilizing a variety of IV iron products demonstrated positive results. The benefits of IV iron used for FID anemia (FIDA) were largely independent of baseline serum iron parameters. 
Table 1. Intravenous iron products studied in clinical trials on chemotherapy-induced anemia (CIA)

\begin{tabular}{lllll}
\hline Product & Trade name & Dose, mg & Infusion & \\
\cline { 4 - 5 } & & & time, min & frequency \\
\hline LMW iron dextran & INFeD $^{\circledR}$ & $100-2,000$ & $60-240$ & Single or divided doses to achieve 1-2 g \\
Iron sucrose & Venofer $^{\circledR}$ & $200-300$ & $5-60$ & Divided doses to achieve $1 \mathrm{~g}$ \\
Ferric gluconate & Ferrlecit $^{\circledR}$ & 125 & 60 & Divided doses to achieve $1 \mathrm{~g}$ \\
Ferric carboxymaltose & Injectafer $^{\circledR}$ & 750 & $7.5-15$ & Single infusion \\
Ferumoxytol & Feraheme $^{\circledR}$ & 510 & $\geq 15$ & Two doses within 1 week \\
Iron isomaltoside & Monofer $^{\circledR}$ & $500-1,000$ & $2-15$ & Single or divided doses to achieve 1-2 g \\
\hline
\end{tabular}

Drug doses listed were those described in CIA clinical trials. LMW, low molecular weight. Ferric carboxymaltose is available as a 750 - $\mathrm{mg}$ vial. Therefore, some clinicians may choose to start with $750 \mathrm{mg}$ and assess response before administering another dose to patients with cancer.

Table 2. Summary of clinical trials on chemotherapy-induced anemia (CIA) using intravenous (IV) iron with erythropoiesis-stimulating agents (ESAs)

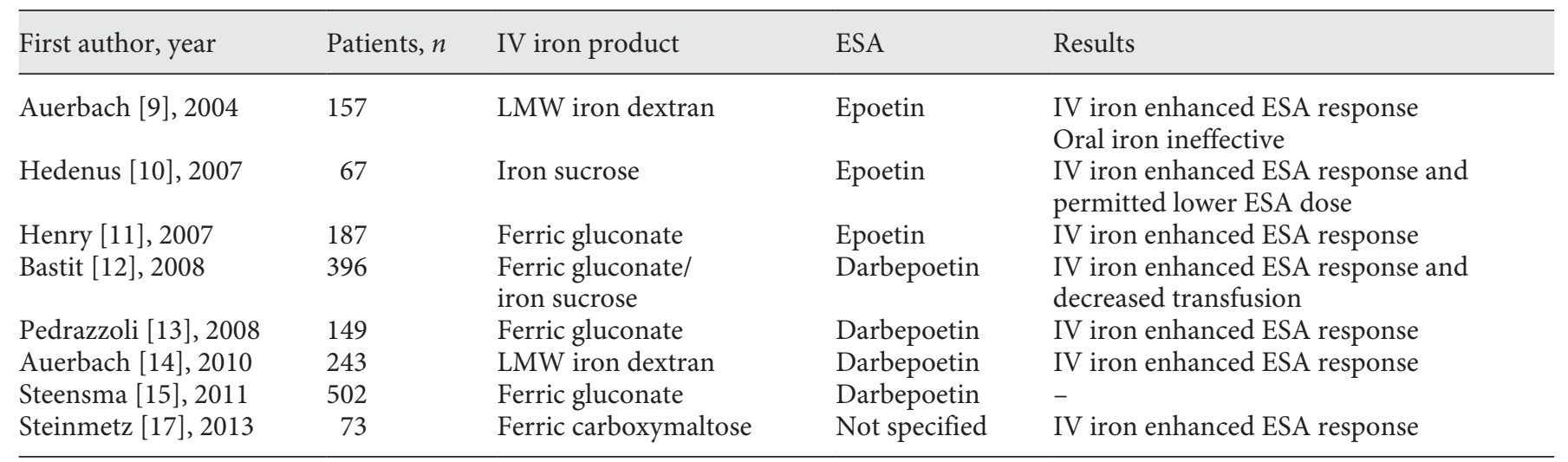

The original publication by Steensma et al. [15] reported no benefit of IV iron in this study. However, a reanalysis of trial data indicated that trial participants who received at least $80 \%$ of the planned IV iron dosage had hematologic responses similar to participants in the other IV iron trials [16]. LMW, low-molecular weight.

\section{Summary of CIA Clinical Trials Using IV Iron Monotherapy}

Although ESAs are useful in treating FID of CIA, their use is associated with potential adverse events and come with myriad restrictions dictated by insurers such as Medicare. For example, ESAs are only approved for cancer patients with incurable diseases, receiving palliative myelosuppressive chemotherapy only, and with $\mathrm{Hb}<10$ $\mathrm{g} / \mathrm{dL}$. Moreover, the most recent label update states that ESAs are "not indicated for patients with cancer receiving myelosuppressive chemotherapy in whom the anemia can be managed by transfusion." As a result of this amendment, it could be argued that ESAs such as darbepoetin alfa and epoetin alfa are no longer indicated for any patient with cancer, or that they are only indicated for a small subset of patients receiving chemotherapy with noncurative intent whose $\mathrm{Hb}$ falls below $10 \mathrm{~g} / \mathrm{dL}$ but remains above any trigger for RBC transfusion. Because few patients with cancer qualify for an ESA, clinicians are in need of alternative therapies to manage anemia beyond RBC transfusion. As of February 2019, 7 CIA clinical trials have investigated IV iron monotherapy; these are summarized in Table 3. Overall, every trial with IV iron monotherapy yielded positive results, which either leads to increased $\mathrm{Hb}$ levels, decreased $\mathrm{RBC}$ transfusion requirement, or both. Using IV iron monotherapy has the advantage of treating both absolute iron deficiency and 
Table 3. Summary of clinical trials on chemotherapy-induced anemia (CIA) using intravenous (IV) iron monotherapy

\begin{tabular}{lcll}
\hline First author, year & Patients, $n$ & IV iron product & Results \\
\hline Kim [18], 2007 & 75 & Iron sucrose & IV iron improved Hb and decreased transfusions \\
Dangsuwan [19], 2010 & 44 & Iron sucrose & IV iron improved Hb and decreased transfusions \\
Abdel-Razeq [20], 2013 & 25 & Iron sucrose & IV iron improved Hb \\
Athibovonsuk [21], 2013 & 64 & Iron sucrose & IV iron decreased transfusions \\
Steinmetz [17], 2013 & 577 & Ferric carboxymaltose & IV iron improved Hb \\
Hedenus [22], 2014 & 17 & Ferric carboxymaltose & IV iron improved Hb \\
Birgegård [23], 2016 & 229 & Iron isomaltoside & IV iron improved Hb \\
Vadhan-Raj [24], 2017 & 75 & Ferumoxytol & IV iron improved Hb \\
\hline
\end{tabular}

$\mathrm{Hb}$, hemoglobin.

FID; also, the patient's cancer status (curable vs. incurable), type of cancer treatment (chemotherapy vs. biologic therapy vs. hormonal therapy), and baseline $\mathrm{Hb} \mathrm{lev-}$ el are not part of required criteria for using IV iron. Thus, more cancer patients with anemia would be eligible for treatment with IV iron monotherapy versus IV iron with ESAs or an ESA alone.

\section{What Do Cancer Society Guidelines Recommend?}

Several cancer society and consensus guideline panels have promulgated recommendations on the use of IV iron in CIA patients. The National Comprehensive Cancer Network (NCCN) guidelines in 2009 recommended IV iron over oral iron therapy in CIA, and subsequent annual updates continue to recommend IV iron $[4,5]$. The European Society for Medical Oncology (ESMO) originally recommended IV iron in their 2010 guideline and confirmed the utility of IV iron in their 2018 update [6]. Another European society (European Organization for the Research and Treatment of Cancer, EORTC) agreed with these recommendations in their 2008 publication [7].

The outlier guideline on this subject has been the American Society of Hematology (ASH)/American Society of Clinical Oncology (ASCO), whose last anemia guideline was in 2010 and did not recommend the use of IV iron in CIA [8]. Unfortunately, the ASH/ASCO guideline has not been updated in many years. The consensus of guidelines that have considered the most recent clinical trial results is that IV iron therapy is recommended to treat CIA.

Intravenous Iron in Cancer- and

Chemotherapy-Induced Anemia

\section{Which Patients Should Receive IV Iron and/or ESAs?}

Iron deficiency can be considered as a spectrum of iron panel laboratory results (Fig. 2). As shown, at the extreme of absolute iron deficiency (ferritin $<30 \mathrm{ng} / \mathrm{mL}$ or a transferrin saturation (TSAT) $<20 \%$, patients would be expected to respond to iron monotherapy. At the opposite extreme of iron repletion (ferritin $\geq 800 \mathrm{ng} / \mathrm{mL}$ and TSAT $\geq 50 \%$ ), anemic patients could be optimally treated with ESA monotherapy should they qualify using the aforementioned criteria. In the intermediate state of FID (ferritin $30-800 \mathrm{ng} / \mathrm{mL}$ or TSAT $20-50 \%$ ), clinicians have 2 options that are supported by clinical trial data in Tables 2 and 3 - IV iron monotherapy or IV iron with ESA. Some physicians and patients may prefer to avoid using ESAs due to the limitations discussed above, as well as the potential risks of ESA therapy. Based upon goals and preferences, IV iron monotherapy may be an appropriate option for these patients. A third option, RBC transfusion, is available and recommended as an appropriate choice by the NCCN guidelines. Physicians should make patients aware of these options and their benefitrisk profiles. Although the risks of ESA therapy are well known, the long-term risks of IV iron therapy in cancer patients are unknown [25].

\section{The Future of IV Iron Is Uncertain}

To date, no IV iron formulation has gained FDA approval specifically for the treatment of patients with cancer. Despite this fact, a common approach to treating FIDA in patients with cancer is to administer parenteral iron with the goal of bypassing the macrophage "iron

Acta Haematol 2019;142:13-20

DOI: $10.1159 / 000496967$ 


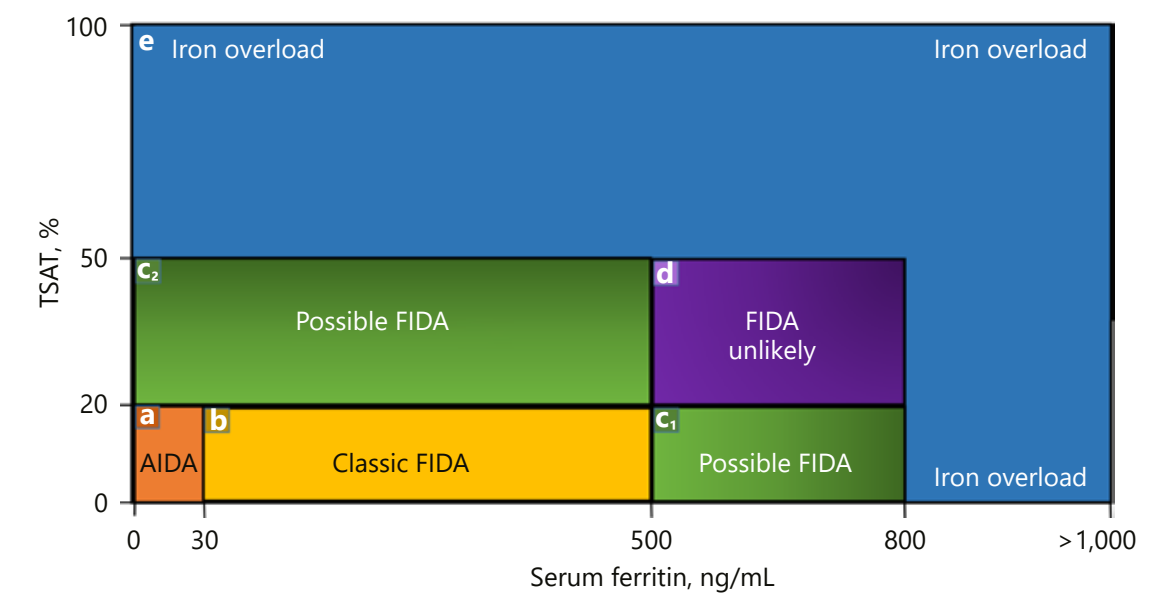

Fig. 2. The relationship between transferrin saturation (TSAT), ferritin, and iron stores, and likelihood of hemoglobin response to iron therapy. a Absolute iron deficiency anemia (AIDA); very responsive to oral or intravenous (IV) iron therapy. These patients should not receive ESAs until iron replete. $\mathbf{b}$ Functional iron deficiency anemia (FIDA); although these patients have normal iron stores, TSAT values are low, indicating iron-restricted erythropoiesis. These patients will likely not respond to oral iron but should respond to IV iron. ESAs should not be used in this group until normal TSAT values are achieved. c Two groups of patients who may have FID: those with increased ferritin levels but low TSAT

block" for a short period of time. As previously mentioned, the long-term consequences of this strategy are largely unknown as most studies assessing the safety of IV iron in cancer patients cease monitoring after 16 weeks, with the exception of one study [23] which followed patients for 6 months. Moreover, the impact of IV iron on tumor growth has been entirely ignored. As ESAs were initially thought to be safe, and doses in clinical trials were escalated, long-term safety concerns surrounding thrombosis, and to a lesser degree poorer survival, in certain populations proved through meta-analyses that "more is not always better." Lessons learned from the ESA era should not be forgotten and must be applied to IV iron as well. Although grossly lacking, prospective studies looking at overall survival in patients with cancer who receive IV iron are sorely needed. Until these data are available, a false sense of security should not obscure or supplant good clinical judgment.

\section{IV vs. Oral Iron}

The study by Birgegård et al. [23], which studied the effect of iron isomaltoside, calls the superiority of the parenteral route into question. Although the $\mathrm{Hb}$ response values $\left(\mathbf{c}_{\mathbf{1}}\right)$ and those with normal TSAT values but lower ferritin levels $\left(\mathbf{c}_{\mathbf{2}}\right)$. Each group per clinical trial data in cancer patients suggests possible responsiveness to IV iron. $\mathbf{d}$ This group of patients has normal TSAT values and elevated ferritin levels and will likely be nonresponsive to IV iron. ESA monotherapy is an option for these patients. e Iron overload state. These patients have elevated serum ferritin levels over $800 \mathrm{ng} / \mathrm{mL}$ and TSAT values over $50 \%$. ESA monotherapy is an option for these patients. Cutoff values for this figure were obtained from the NCCN guideline (version 3.2018) $[4,5]$.

was similar between the oral and IV iron groups, IV iron was associated with a more rapid $\mathrm{Hb}$ response and more rapid iron repletion. This is in contrast to the findings by Auerbach et al. [9], who found IV iron to be superior to oral iron in terms of magnitude of $\mathrm{Hb}$ increase. More studies are needed in patients with varying degrees of FIDA to determine who will or will not respond to oral iron.

\section{Novel Approaches}

If more iron is not the answer or perhaps not an option, new approaches designed to make endogenous sequestered iron more bioavailable may provide a valuable alternative solution. Targeting the hepcidin pathway is most logical as iron is primarily sequestered in the spleen within the reticuloendothelial system. Macrophages within the reticuloendothelial system lack ferroportin as a result of an overabundance of hepcidin. Through either inhibiting the production of hepcidin or limiting its biological activity through neutralization, ferroportin receptors could flourish allowing macrophages to regain their ability to export iron. It has long been known that coadministration of oral iron with ascorbic acid (vitamin C)
18

Acta Haematol 2019;142:13-20 DOI: $10.1159 / 000496967$
Rodgers/Gilreath 
enhances iron absorption [26]; however, few publications have investigated the mechanism by which this occurs. Dietary iron is absorbed by the divalent metal transporter DMT [27]. The most widely accepted hypothesis is that ascorbic acid reduces ferric iron $\left(\mathrm{Fe}^{3+}\right)$ to the bioavailable form ferrous iron $\left(\mathrm{Fe}^{2+}\right)$, but data to support this as the sole mechanism for increased iron absorption are lacking. Other possibilities include a role for ascorbic acid in hepcidin synthesis. Interestingly, Chiu et al. [28] showed in an in vitro model that ascorbic acid has the ability to modify hepcidin mRNA gene transcription. Whether vitamin $\mathrm{C}$ has the ability to fully downregulate hepcidin production in vivo remains to be determined. Clinical studies assessing the impact of IV ascorbic acid in patients with chronic kidney diseases and FIDA have been conducted. Increases in $\mathrm{Hb}$, with or without ESA use, have been noted [29]. Presumably, the mechanism by which ascorbic acid allowed for iron mobilization lies in its ability to decrease hepcidin production. Other possible targets aim to modify components of the hepcidin synthesis pathway through augmentation of SMAD signaling, which is regulated by many components, including transferrin receptor 1 and 2, hemojuvelin, BMP-2, BMP-6, matriptase, human hemochromatosis protein (HFE), and others $[2,30]$. Modification of these targets remains to be tested clinically in patients with cancer.

Ultimately, a more complete understanding of iron metabolism will hopefully lead to the development of novel erythropoietic adjuncts to help ameliorate FIDA and improve quality of life without compromising outcome.

\section{Statement of Ethics}

Not applicable.

\section{Disclosure Statement}

The authors do not have financial affiliation or involvement with any organization relating to the subject matter discussed in this paper.

\section{References}

1 Gilreath JA, Stenehjem DD, Rodgers GM. Diagnosis and treatment of cancer-related anemia. Am J Hematol. 2014 Feb;89(2):203-12.

2 Andrews NC. Forging a field: the golden age of iron biology. Blood. 2008 Jul;112(2):21930.

3 Kautz L, Jung G, Valore EV, Rivella S, Nemeth E, Ganz T. Identification of erythroferrone as an erythroid regulator of iron metabolism. Nat Genet. 2014 Jul;46(7):678-84.

4 Rodgers GM. A perspective on the evolution of management of cancer- and chemotherapy-induced anemia. J Natl Compr Canc Netw. 2012 Apr;10(4):434-7.

5 Rodgers GM 3rd, Becker PS, Blinder M, Cella D, Chanan-Khan A, Cleeland C, et al. Cancerand chemotherapy-induced anemia. J Natl Compr Canc Netw. 2012 May;10(5):628-53.

6 Aapro M, Beguin Y, Bokemeyer C, Dicato M, Gascón P, Glaspy J, et al; ESMO Guidelines Committee. Management of anaemia and iron deficiency in patients with cancer: ESMO Clinical Practice Guidelines. Ann Oncol. 2018 Oct;29 Suppl 4:iv96-110.

7 Aapro MS, Link H. September 2007 update on EORTC guidelines and anemia management with erythropoiesis-stimulating agents. Oncologist. 2008;13 Suppl 3:33-6.
8 Rizzo JD, Brouwers M, Hurley P, Seidenfeld J, Arcasoy MO, Spivak JL, et al; American Society of Hematology and the American Society of Clinical Oncology Practice Guideline Update Committee. American Society of Clinical Oncology/American Society of Hematology clinical practice guideline update on the use of epoetin and darbepoetin in adult patients with cancer. Blood. 2010 Nov 18;116(20): 4045-59.

9 Auerbach M, Ballard H, Trout JR, McIlwain $\mathrm{M}$, Ackerman A, Bahrain $\mathrm{H}$, et al. Intravenous iron optimizes the response to recombinant human erythropoietin in cancer patients with chemotherapy-related anemia: a multicenter, open-label, randomized trial. J Clin Oncol. 2004 Apr;22(7):1301-7.

10 Hedenus M, Birgegård G, Näsman P, Ahlberg L, Karlsson T, Lauri B, et al. Addition of intravenous iron to epoetin beta increases hemoglobin response and decreases epoetin dose requirement in anemic patients with lymphoproliferative malignancies: a randomized multicenter study. Leukemia. 2007 Apr;21(4): 627-32.

11 Henry DH, Dahl NV, Auerbach M, Tchekmedyian S, Laufman LR. Intravenous ferric gluconate significantly improves response to epoetin alfa versus oral iron or no iron in anemic patients with cancer receiving chemotherapy. Oncologist. 2007 Feb;12(2):231-42.
12 Bastit L, Vandebroek A, Altintas S, Gaede B, Pintér T, Suto TS, et al. Randomized, multicenter, controlled trial comparing the efficacy and safety of darbepoetin alpha administered every 3 weeks with or without intravenous iron in patients with chemotherapy-induced anemia. J Clin Oncol. 2008 Apr;26(10): 1611-8.

13 Pedrazzoli P, Farris A, Del Prete S, Del Gaizo F, Ferrari D, Bianchessi C, et al. Randomized trial of intravenous iron supplementation in patients with chemotherapy-related anemia without iron deficiency treated with darbepoetin alpha. J Clin Oncol. 2008 Apr;26(10): 1619-25.

14 Auerbach M, Silberstein PT, Webb RT, Averyanova S, Ciuleanu TE, Shao J, et al. Darbepoetin alfa 300 or $500 \mu$ g once every 3 weeks with or without intravenous iron in patients with chemotherapy-induced anemia. Am J Hematol. 2010 Sep;85(9):655-63.

15 Steensma DP, Sloan JA, Dakhil SR, Dalton R, Kahanic SP, Prager DJ, et al. Phase III, randomized study of the effects of parenteral iron, oral iron, or no iron supplementation on the erythropoietic response to darbepoetin alfa for patients with chemotherapy-associated anemia. J Clin Oncol. 2011 Jan;29(1):97105. 
16 Steensma DP, Sloan JA, Loprinzi CL. Reply to M. Aapro et al. J Clin Oncol. 2011;29(17): e527-8.

17 Steinmetz T, Tschechne B, Harlin O, Klement B, Franzem M, Wamhoff J, et al. Clinical experience with ferric carboxymaltose in the treatment of cancer- and chemotherapy-associated anaemia. Ann Oncol. 2013 Feb;24(2): 475-82.

18 Kim YT, Kim SW, Yoon BS, Cho HJ, Nahm EJ, Kim SH, et al. Effect of intravenously administered iron sucrose on the prevention of anemia in the cervical cancer patients treated with concurrent chemoradiotherapy. Gynecol Oncol. 2007 Apr;105(1):199-204.

19 Dangsuwan P, Manchana T. Blood transfusion reduction with intravenous iron in gynecologic cancer patients receiving chemotherapy. Gynecol Oncol. 2010 Mar;116(3):522-5.

20 Abdel-Razeq H, Abbasi S, Saadi I, Jaber R, Abdelelah $\mathrm{H}$. Intravenous iron monotherapy for the treatment of non-iron-deficiency anemia in cancer patients undergoing chemotherapy: a pilot study. Drug Des Devel Ther. 2013 Aug; 7:939-44.
21 Athibovonsuk P, Manchana T, Sirisabya N. Prevention of blood transfusion with intravenous iron in gynecologic cancer patients receiving platinum-based chemotherapy. Gynecol Oncol. 2013 Dec;131(3):679-82.

22 Hedenus M, Karlsson T, Ludwig H, Rzychon B, Felder M, Roubert B, et al. Intravenous iron alone resolves anemia in patients with functional iron deficiency and lymphoid malignancies undergoing chemotherapy. Med Oncol. 2014 Dec;31(12):302.

23 Birgegård G, Henry D, Glaspy J, Chopra R, Thomsen LL, Auerbach M. A randomized noninferority trial of intravenous iron isomaltoside versus oral iron sulfate in patients with nonmyeloid malignancies and anemia receiving chemotherapy: the PROFOUND Trial. Pharmacotherapy. 2016 Apr;36(4): 402-14.

24 Vadhan-Raj S, Dahl NV, Bernard K, Li Z, Strauss WE. Efficacy and safety of IV ferumoxytol for iron deficiency anemia in patients with cancer. J Blood Med. 2017 Dec;8:199209.
25 Gilreath JA, Stenehjem DD, Rodgers GM. Total dose iron dextran infusion in cancer patients: is it SaFe2+? J Natl Compr Canc Netw. 2012 May;10(5):669-76.

26 Hallberg L, Brune M, Rossander-Hulthén L. Is there a physiological role of vitamin $\mathrm{C}$ in iron absorption? Ann N Y Acad Sci. 1987;498: 324-32.

27 Gunshin H, Mackenzie B, Berger UV, Gunshin Y, Romero MF, Boron WF, et al. Cloning and characterization of a mammalian protoncoupled metal-ion transporter. Nature. 1997 Jul;388(6641):482-8.

28 Chiu PF, Ko SY, Chang CC. Vitamin C affects the expression of hepcidin and erythropoietin receptor in HepG2 cells. J Ren Nutr. 2012 May;22(3):373-6.

29 Einerson B, Chaiyakunapruk N, Kitiyakara C, Maphanta S, Thamlikitkul V. The efficacy of ascorbic acid in suboptimal responsive anemic hemodialysis patients receiving erythropoietin: a meta-analysis. J Med Assoc Thai. 2011 Feb;94 Suppl 1:S134-46.

30 Frazer DM, Anderson GJ. Iron imports. I. Intestinal iron absorption and its regulation. Am J Physiol Gastrointest Liver Physiol. 2005 Oct;289(4):G631-5. 